York and London, 1963), has seen a flood of research into the solarterrestrial environment. Accumulation of space observations, and of theoretical work stimulated by these data, has been increasingly demanding release through a new synthesis of the same ilk as Parker's. A. J. Hundhausen's new book satisfies this need in such a concise and lucid manner as to be equally suitable as an undergraduate text and as a research reference manual.

After a historical introduction, invaluable to non-specialists, Hundhausen classifies solar wind phenomena, identifying observed structures with characteristic theoretical time scales in the mag. netized plasma. This provides a basis for the main themes of mathematical modelling of large scale phenomena in steady structureless flow of the wind and, subsequently, of non-steady flow in structured features.

Devotion of almost half the book to the steady flow problem itself indicates the "plethora of ... partially proven theories" proposed to overcome the discrepancies between both one- and twofluid wind models and observations. Though the author remarks, in typical style, that ". . . unique conclusions regarding the physical processes [which resolve this problem] . . can be deduced only by true believers ...", he succeeds admirably in laying foundations for future work by sifting the morass of free parameters and coronal boundary conditions variously adopted in models.

Following a chapter on the role of helium and heavier elements in the steady wind, structure is introduced into the flow and it is at once evident that high speed plasma streams superposed on the quiet wind are the rule rather than the exception. The apparent transience of such streams is emphasized as due to the confinement of rapid plasma flow within magnetic sectors co-rotating with the Sun and their theoretical description is developed in this framework. Correlation of these stream sectors with the large scale organization of the solar magnetic field, rather than with active regions, appears as one of the most important results of spacecraft observations.

Severe transient solar wind disturb. ances in the form of flare-driven shocks provide the last theme and exemplify the improvement of data in the last decade - for example, a reduction from 100 to about 2 in the uncertainty factor in blast-wave mass estimates. Seeking to find some small fault in such an excellent book on a wide topic, one might consider incompleteness or typographical errors. Near total absence of the latter and coverage of omissions in a final bibliographic chapter, however, allow only an unqualified recommendation of the monograph.

JOHN C. BROWN

\section{Nuclear Interactions}

Fundamental Interactions and the Nucleus. By R. J. Blin-Stoyle. Pp. $x v+345$. (North-Holland: Amsterdam and London ; American Elsevier: New York, 1973.) Dfl. $60 ; \$ 21$.

FOR many years Professor Blin-Stoyle and his colleagues have made detailed studies of the manifestation of weak, electromagnetic and T-violating interactions in the nucleus. Now he has embodied the results of his studies in a comprehensive book that will be welcomed by all nuclear physicists.

After a short introductory chapter on fundamental interactions and the nucleus, the following four chapters (107 pages) are devoted to nuclear beta decay including a detailed consideration of the polar and axial vector currents, unusual hadron currents and interactions, and the lepton current and the neutrino. The next two chapters consider muon capture (27 pages) and the weak interaction potential (14 pages). The weak-interaction aspects of nuclear reactions follow, with chapters on the effect of a parity-violating potential in low energy processes, especially in alpha decay and in electromagnetic transitions (30 pages), and on time reversal invariance in nuclear reactions and in electromagnetic transitions (37 pages). Various electromagnetic and charge-dependent interactions are considered next (40 pages), with sections on the nuclear electromagnetic Hamiltonian, charge dependence of the inter-nucleon potential, magnetic dipole exchange effects and isospin multiplet energies, with a brief note on isospin in nuclear reactions. The last chapter is a very brief (8 pages) account of strong interactions in the nucleus. There are useful appendices on notation and conventions, relativistic and non-relativistic quantum mechanics, quantized fields, and isospin, charge symmetry and G-parity.

From this summary it will be clear that the scope of this book is much narrower than the title might indicate, and that it is concerned essentially with weak interactions and the nucleus, and even then there is no mention of electron scattering and muonic $\mathrm{X}$-rays and the knowledge that can be obtained from them of the nuclear charge distribution. There is no account of the way the nuclear properties can be calculated from the nucleon-nucleon interaction or of kaon capture. The book is thus concerned essentially with the ways that various nuclear phenomena can be used to obtain more precise information about the fundamental weak nuclear interactions. As such, it is a very comprehensive and thorough review of a difficult field.

The need to make frequent use of field and group theoretical concepts and relativistic dynamics does not make for easy reading, and although it is impossible to develop these $a b$ initio some more help could have been given to the less well-prepared reader by references to standard textbooks and review articles. Apart from this, the theoretical background is clearly described, with derivations of the formulae needed to interpret the experimental data.

Many of the most important investigations in this field, such as the determination of the parity-violating potential in nuclear reactions, the charge dependence of the internucleon potential and the detection of $\mathrm{T}$-invariant second class currents in beta decay, require extremely precise experiments and very careful evaluation of all possible correction terms. Such work is comprehensively described with detailed and up-to-date references to the original work, and clear tables showing the present status of the problems. For this alone it will be invaluable to all research workers in the field.

There is a full list of references at the end. It would have been helpful to include an index of author and a fuller subject index.

P. E. HodGSON

\section{Synthetic Method}

Advanced Organic Synthesis. By Richard S. Monson. Pp. xii +203. (Academic: New York and London, June 1971.) $\$ 15 ; £ 7$.

WRITING books on chemical synthesis seems to have become as popular (though hardly as rewarding) an occupation as actually working at it, with several recent examples ranging from analysis of strategy, through systematic listings of functional group transformations, to descriptive accounts of the nuts and bolts of the subject. Dr Monson's book fits firmly into the latter category. It contains detailed procedures for the preparation of 250 and more organic compounds, the vast majority of them being uncomplicated, one-step operations. In this respect the book is very similar in style to Organic Synthesis, though it cannot match the scope and detail achieved by fifty-two volumes of the latter. In fact, many of the procedures are taken directly from Organic Synthesis, to which the author has added a few general introductory remarks. The experiments are chosen so as to exemplify the following processes: oxidation; hydride and related reductions ; dissolving metal reductions; hydroboration; catalytic hydrogenation; introduction of halogen; miscellaneous elimination, substitution and addition reactions; the Diels-Alder reaction; enamines ; enolates; the Wittig reaction; reactions of 